\title{
Birth Weight and Adult Obesity Index in Relation to the Risk of \\ OPEN ACCESS \\ Edited by: \\ Soo-Kyoung Choi, \\ Hypertension: A Prospective Cohort Study in the UK Biobank
} Yonsei University College of Medicine,

South Korea

Reviewed by:

Jane A. Leopold,

Brigham and Women's Hospital and Harvard Medical School,

United States

Elise Peery Gomez-Sanchez, University of Mississippi Medical

Center, United States

*Correspondence:

Dong Hang hangdong@nimu.edu.cn

Wei Zhao

zhaowei0714@nicemice.cn

Aihua Gu

aihuagu@njmu.edu.cn

tThese authors have contributed equally to this work and share first

authorship

FThese authors have contributed equally to this work and share last authorship

Specialty section:

This article was submitted to

Hypertension,

a section of the journal

Frontiers in Cardiovascular Medicine

Received: 03 December 2020

Accepted: 12 May 2021

Published: 17 June 2021

Citation:

Zhang Y, Liang J, Liu Q, Fan X, Xu C,

Gu A, Zhao W and Hang D (2021) Birth Weight and Adult Obesity Index

in Relation to the Risk of Hypertension:

A Prospective Cohort Study in the UK Biobank.

Front. Cardiovasc. Med. 8:637437.

doi: 10.3389/fcvm.2021.637437

\author{
Yi Zhang ${ }^{1 \dagger}$, Jingjia Liang $^{1 \dagger}$, Qian Liu ${ }^{1 \dagger}$, Xikang Fan ${ }^{2}$, Cheng Xu ${ }^{1}$, Aihua Gu ${ }^{1 * \neq}$, Wei Zhao ${ }^{3 * \neq}$ \\ and Dong Hang ${ }^{2 * \neq}$ \\ ${ }^{1}$ State Key Laboratory of Reproductive Medicine, Institute of Toxicology, Nanjing Medical University, Nanjing, China, \\ ${ }^{2}$ Department of Epidemiology and Biostatistics, Jiangsu Key Lab of Cancer Biomarkers, Prevention and Treatment, \\ Collaborative Innovation Center for Cancer Personalized Medicine, School of Public Health, Nanjing Medical University, \\ Nanjing, China, ${ }^{3}$ Jinling Hospital Department of Reproductive Medical Center affiliated School of Medicine, Nanjing \\ University, Nanjing, China
}

Objectives: To investigate the association between birth weight and the risk of hypertension, and to examine the interaction between birth weight and the adult obesity index.

Methods: We included 199,893 participants who had birth weight data and no history of hypertension at baseline (2006-2010) from the UK Biobank. A multivariate cubic regression spline was used to visually explore the dose-response relationship. Multivariate Cox proportional hazard regression models were used to calculate hazard ratios (HRs) and 95\% confidence intervals (Cls).

Results: We observed a nonlinear inverse association between birth weight and hypertension. The risk for hypertension decreased as birth weight increased up to approximately $3.80 \mathrm{~kg}$. Compared with the participants with the fourth quintile of birth weight $(3.43-3.80 \mathrm{~kg})$, those with the first quartile of birth weight $(<2.88 \mathrm{~kg})$ were associated with a $25 \%$ higher risk of hypertension [HR 1.25; 95\% Cl (1.18-1.32)]. In addition, the participants with birth weight $<2.88 \mathrm{~kg}$ and body mass index $\geq 30 \mathrm{~kg} / \mathrm{m}^{2}$ had the highest risk [HR 3.54; 95\% Cl (3.16-3.97); $p$ for interaction $<0.0001]$, as compared with those with birth weight between $3.43-3.80 \mathrm{~kg}$ and body mass index between $18.5-25.0 \mathrm{~kg} / \mathrm{m}^{2}$. These associations were largely consistent in the stratified and sensitivity analyses.

Conclusion: Our findings indicate that lower birth weight is nonlinearly correlated with higher risk of hypertension, and birth weight between $3.43-3.80 \mathrm{~kg}$ might represent an intervention threshold. Moreover, lower birth weight may interact with adult obesity to significantly increase hypertension risk.

Keywords: birth weight, adult obesity index, hypertension, blood pressure, epidemiology, prospective study 


\section{INTRODUCTION}

Fetal malnutrition, the primary indicator of which is low birth weight, can permanently alter organ structure and function in a way that predisposes the offspring to cardiovascular disease (CVD) in adulthood $(1,2)$. Growing evidence has suggested that low birth weight increases the risk of hypertension (3-5).

Although some epidemiological studies have suggested a nonlinear inverse association between birth weight and hypertension (6-9), the intervention threshold of birth weight for hypertension remains undetermined. A previous metaanalysis including 4,335,149 participants suggested that those with birth weight of $4.0-4.5 \mathrm{~kg}$ had the lowest risk (10). However, the meta-analysis contained significant heterogeneity that limited the validity of summary estimates, and the ability to control for potential confounders (e.g., maternal smoking) was insufficient. In addition, previous results are mixed regarding the association between high birth weight and hypertension risk. Some studies reported higher birth weight in relation to increased hypertension risk (11-13), whereas other studies did not find such an association $(14,15)$. These conflicting results might be attributed to the differences in sample sizes and confounder adjustments (13). Furthermore, low birth weight and obesity in adulthood can both stimulate the sympathetic nervous system and alter renal function $(16,17)$. Few studies have examined potential interaction between birth weight and the adult obesity index on hypertension risk, although there is evidence showing an effect on CVD risk $(18,19)$.

Therefore, for the current study we used data from the UK Biobank, a large prospective cohort study conducted in UK, to investigate the dose-response association of birth weight with hypertension and blood pressure. Moreover, we examined potential interactions between birth weight and the adult obesity index.

\section{METHODS AND MATERIALS}

\section{Study Population}

The UK Biobank is a prospective cohort study recruiting half a million participants aged 37-73 years between 2006 and 2010 (20). At baseline, participants were asked to provide electronically signed consent, answer touch-screen questionnaires, and complete physical and anthropometric measurements. Follow-up with the UK Biobank was performed on an ongoing basis through study visits and linkage to national health records, death registers, and primary care records, as described previously (21). The UK Biobank has received ethical approvals from the UK Biobank Research Ethics Committee and Human Tissue Authority.

In the current study, we excluded participants who self-reported a history of hypertension or were taking antihypertensive medication at baseline $(n=147,989)$. We

Abbreviations: BMI, body mass index; CVD, cardiovascular disease; WHR, waistto-hip ratio; SBP, systolic blood pressure; DBP, diastolic blood pressure; LRT, likelihood ratio test; MET, metabolic equivalent of task; SD, standard deviation; HR, hazard ratio; CI, confidence interval; Ref, reference; NA, not applicable. further excluded 151,078 individuals with missing birth weight data and 3,661 individuals with missing values on the main covariables. Finally, a total of 199,893 participants were included (Supplementary Figure 1). The basic characteristics of the excluded participants were similar to those included (Supplementary Table 1).

\section{Birth Weight and Physical Measurements}

Participants were asked to report their own birth weight (either in kilograms directly, or in imperial pounds and ounces). Standing height was measured using a Seca202 device. Body mass index (BMI) was defined as weight divided by height squared $\left(\mathrm{m}^{2}\right)$. Waist and hip circumferences were measured with a Seca200 measuring tape using standard procedures, and waistto-hip ratio (WHR) was the ratio of waist circumference to hip circumference.

Systolic blood pressure (SBP) and diastolic blood pressure (DBP) measurements were taken in a seated position after a few minutes of rest using an Omron 705 IT electronic blood pressure monitor. A manual sphygmometer was used if the standard automated device could not be employed. Further details of these measurements can be found in the UK Biobank online protocol (https://biobank.ndph.ox.ac.uk/showcase/ showcase/docs/Bloodpressure.pdf" https://biobank.ndph.ox.ac. uk/showcase/showcase/docs/Bloodpressure.pdf). Means of SBP and DBP from two automated or two manual blood pressure measurements were calculated.

\section{Assessment of Hypertension}

The date and cause of hospital admissions were confirmed by electronic health records linkage to health episode statistics (England and Wales) and Scottish morbidity records (Scotland). Date of death was obtained from death certificates held by the National Health Service Information Center (England and Wales) and the National Health Service Central Register Scotland (Scotland) (22). The primary outcome was incident hypertension, which was defined according to the International Classification of Diseases edition 10 (ICD-10) codes I10-I15.

\section{Statistical Analysis}

Person-time of follow-up was calculated for each participant from the age in months at the return date of the baseline questionnaire (2006-2010) until the age in months at the date of first diagnosis of hypertension or the end of followup (November 30, 2016, in Edinburgh, Scotland, and January 31, 2018, in England or Wales), whichever came first. Cox proportional hazards regression models with age as the time scale were used to analyze the association between birth weight and hypertension, reporting hazard ratio (HR) and 95\% confidence intervals $(\mathrm{CI})$.

A multivariate restricted cubic spline with four knots was used to visually explore the nonlinear association of birth weight with hypertension. We used a likelihood ratio test (LRT) to compare the model with only the linear term of birth weight to the model with both the linear and the cubic spline terms, with a $p$-value $<0.05$ denoting significant nonlinearity. 
In the multivariate analysis of hypertension, we adjusted for age, sex, and early life events, including maternal smoking, breastfeeding, part of a multiple birth, and birthplace (Model 1). We also further adjusted for the Townsend deprivation index, college or university degree, BMI, summed metabolic equivalent of task hours per week (MET-hours/week) for physical activity, smoking status, alcohol intake frequency, intake of vegetables and fruit, family history of hypertension, and prevalence of diabetes (Model 2). Multivariate linear regression analysis was conducted to examine the associations between birth weight and SBP and DBP separately $(n=199,765)$. Birth weight was treated as a categorical (the quintiles) or continuous (per SD increment) variable.

Stratified analyses were conducted according to sex (male, female), maternal smoking (yes, no), breastfeeding (yes, no), physical activity (<median, $\geq$ median), and smoking status (never, former, current). We tested the interaction between birth weight and each of the stratification variables using LRT, comparing a model with and without interaction terms.

In the joint analysis of birth weight and BMI, the fourth quintile of birth weight $(3.43-3.80 \mathrm{~kg})$ and BMI in adulthood between $18.5-25.0 \mathrm{~kg} / \mathrm{m}^{2}$ was treated as the reference group. $P$ for interaction was assessed by a Wald test for the cross-product terms between birth weight and BMI (continuous). Sensitivity analyses were performed by excluding individuals who were part of multiple births $(n=5,431)$.

All statistical tests were two-sided, and SAS version 9.4 (SAS Institute) was used for all analyses. $P<0.05$ was defined as statistically significant.

\section{RESULTS}

During a median follow-up of 8.8 (interquartile range: 8.19.4) years, 12,333 hypertension events occurred among 199,893 participants. The basic characteristics of participants according to the quintile of birth weight are shown in Table 1 . Those with lower birth weight were more likely to be part of a multiple birth and exposed to maternal smoking around birth, and were less likely to be breastfed.

A nonlinear inverse association between birth weight and hypertension is observed in Figure 1. The risk of hypertension decreased as birth weight increased up to approximately $3.80 \mathrm{~kg}$ ( $p$ for nonlinearity $=0.0004$ ).

In the fully adjusted models, compared with the fourth quintile of birth weight $(3.43-3.80 \mathrm{~kg})$, the first quartile $(<2.88 \mathrm{~kg})$ was associated with a $25 \%$ higher risk of hypertension [HR 1.25; 95\% CI (1.18-1.32)] (Table 2).

Table 3 shows the association between birth weight and blood pressure. A unit increase per SD increment in birth weight was associated with lower SBP $[\beta$ coefficient $=-1.07 ; 95 \%$ CI $(-1.14$ to -1.00$)]$ and lower DBP $[\beta$ coefficient $=-0.47 ; 95 \% \mathrm{CI}(-0.51$ to -0.44$)$ ].

In the stratified analysis (Supplementary Figure 2), the association between birth weight and hypertension was largely consistent across subgroups, except that the association was stronger in females $(p$ for interaction $=0.004$ ).
A multiplicative interaction between birth weight and obesity index was observed in the joint analysis for birth weight and adult BMI in relation to hypertension risk (Figure 2). Compared with the reference group (birth weight between $3.43-3.80 \mathrm{~kg}$ and BMI between $18.5-25 \mathrm{~kg} / \mathrm{m}^{2}$ ), participants with low birth weight and adult obesity (birth weight $<2.88 \mathrm{~kg}$ and $\mathrm{BMI} \geq 30 \mathrm{~kg} / \mathrm{m}^{2}$ ) had the highest risk of hypertension [HR 3.54; 95\% CI (3.16, $3.97)$; $p$ for interaction $<0.0001]$. A similar pattern of correlations was observed between birth weight, WHR, and hypertension (Supplementary Figure 3).

Sensitivity analysis by excluding 5,431 participants who were part of a multiple birth showed similar associations between birth weight and hypertension (Supplementary Table 2).

\section{DISCUSSION}

In this large prospective cohort study, we observed a nonlinear association between birth weight and hypertension risk. Moreover, we observed a multiplicative interaction between birth weight and obesity index, and participants with low birth weight and adult obesity had the highest risk of hypertension.

Most previous studies investigating the association between birth weight and hypertension risk were based on predefined categories of birth weight. The lowest risk for hypertension was observed in different ranges of birth weight, such as $2.5-3.5 \mathrm{~kg}$, $\geq 4.5 \mathrm{~kg}, 4.0-4.5 \mathrm{~kg}$, and $\geq 4.0 \mathrm{~kg}(5,6,10,23)$. A multivariate restricted cubic spline can display more information than the categorical data, and in the current study a nonlinear inverse association was found between birth weight and hypertension. The risk of hypertension decreased as birth weight increased, up to approximately $3.80 \mathrm{~kg}$. Our data indicated that higher birth weight did not increase the hypertension risk, in line with the results from previous studies $(14,24)$. The World Health Organization defined low birth weight as $<2.50 \mathrm{~kg}$ (25). The cutoff was based on epidemiological observations that infants with birth weight $<2.50 \mathrm{~kg}$ had a higher mortality risk than $\geq 2.50 \mathrm{~kg}$ (26). Nevertheless, it may not be the optimal target for mitigating the increasing epidemic of hypertension. Our findings suggested that birth weight between $3.43-3.80 \mathrm{~kg}$ might represent a potential threshold for reducing hypertension risk.

Epidemiological evidence has shown an association between low birth weight and obesity in adulthood (27). Consistently, we found that individuals with lower birth weight were more likely to have a higher BMI or waist-to-hip ratio in the UK biobank (data not shown). When we adjust for potential confounders, including BMI or waist-to-hip ratio, the association between birth weight and hypertension remained stable. We also found an inverse association between birth weight and blood pressure, in line with the results from previous observational studies $(3,28$, 29). Furthermore, a Mendelian randomization study, which took advantage of genetic variants as instrumental variables for birth weight, supported an inverse association between birth weight and blood pressure (30). However, several studies reported that birth weight was inversely associated with SBP only $(31,32)$. This might be attributed to the limited statistical power of these studies to identify the relatively small effects of birth weight on 
TABLE 1 | Basic characteristics of study participants according to birth weight.

\begin{tabular}{|c|c|c|c|c|c|}
\hline \multirow[t]{2}{*}{ Characteristics } & \multicolumn{5}{|c|}{ Birth weight quintiles } \\
\hline & Q1 (<2.88kg) & Q2 (2.88-3.18kg) & Q3 (3.19-3.42kg) & Q4 (3.43-3.80 kg) & Q5 (>3.80 kg) \\
\hline Participants, No. (\%) & $39,042(19.53)$ & $43,780(21.90)$ & $36,948(18.48)$ & 40,493 (20.26) & $39,630(19.83)$ \\
\hline \multicolumn{6}{|l|}{ Perinatal } \\
\hline Female, No. (\%) & $28,740(73.61)$ & 29,709 (67.86) & 23,790 (64.39) & 23,623 (58.34) & $20,757(52.38)$ \\
\hline White, No. (\%) & 37,252 (95.70) & $42,165(96.56)$ & $36,104(97.92)$ & 39,399 (97.52) & $38,674(97.80)$ \\
\hline Part of a multiple birth, No. (\%) & $3,880(9.95)$ & $806(1.84)$ & $366(0.99)$ & $228(0.56)$ & $151(0.38)$ \\
\hline Breastfeeding, No. (\%) & 20,987 (60.98) & $27,614(69.76)$ & $24,015(70.23)$ & $26,538(71.44)$ & $25,581(72.49)$ \\
\hline Maternal smoking around birth, No. (\%) & 11,988 (34.32) & 11,577 (29.33) & $9,167(27.43)$ & $9,260(25.26)$ & $8,843(24.83)$ \\
\hline \multicolumn{6}{|l|}{ Baseline } \\
\hline Age, mean (SD), year & $54.32(8.09)$ & $54.32(8.13)$ & $53.71(7.87)$ & $53.30(7.96)$ & $54.41(8.13)$ \\
\hline Townsend deprivation index <0, No. (\%) & $28,312(72.52)$ & $32,683(74.65)$ & $27,611(74.73)$ & $30,302(74.83)$ & $29,517(74.48)$ \\
\hline BMI, mean (SD), kg/m² & $26.39(4.57)$ & $26.25(4.35)$ & $26.39(4.34)$ & $26.60(4.40)$ & $27.06(4.49)$ \\
\hline Physical activity, mean (SD), MET-hours/week & $44.85(44.71)$ & $44.62(44.09)$ & $44.57(44.23)$ & $44.26(44.52)$ & $45.79(46.00)$ \\
\hline Current smoking, No. (\%) & $3,985(10.24)$ & $4,246(9.72)$ & $3,875(10.52)$ & $4,116(10.19)$ & 4,325 (10.95) \\
\hline Alcohol intake daily or almost daily, No. (\%) & $6,291(16.12)$ & $8,360(19.11)$ & $7,313(19.81)$ & $8,157(20.16)$ & $8,421(21.26)$ \\
\hline Family history of hypertension, No. (\%) & $19,139(52.42)$ & $21,099(51.13)$ & $18,251(52.08)$ & $19,469(50.89)$ & $18,341(49.40)$ \\
\hline Vegetable intake, mean (SD), serving/day & $2.25(2.13)$ & $2.23(2.14)$ & $2.24(2.09)$ & $2.23(2.08)$ & $2.23(2.21)$ \\
\hline Fruit intake, mean (SD), serving/day & $2.24(1.57)$ & $2.23(1.51)$ & $2.24(1.53)$ & $2.23(1.54)$ & $2.25(1.57)$ \\
\hline DBP, mean (SD), mmHg & $80.89(9.74)$ & 80.52 (9.68) & $80.57(9.60)$ & $80.50(9.60)$ & $80.73(9.57)$ \\
\hline SBP, mean (SD), mmHg & $134.32(17.94)$ & $133.48(17.60)$ & 132.89 (17.06) & $132.42(17.01)$ & $133.24(16.93)$ \\
\hline
\end{tabular}

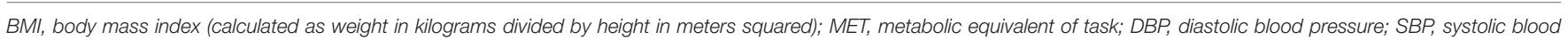
pressure; $S D$, standard deviation.

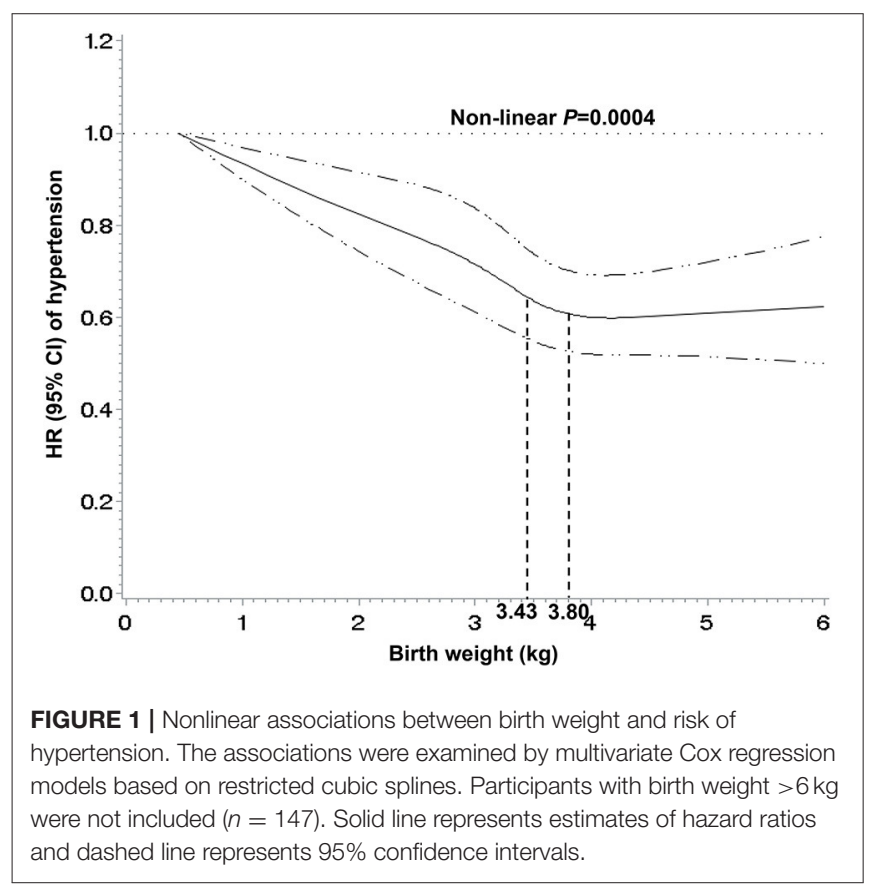

DBP (31). The current study confirmed that the magnitude of the association for DBP was smaller than that for SBP.

Joint analysis indicated that participants with low birth weight and adult obesity had the highest risk of hypertension. Consistently, previous studies have shown that low-birth-weight children with obesity now tend to have higher values of systolic blood pressure than those who are obese with normal birth weight $(33,34)$. It is plausible that individuals with an abnormal intrauterine environment (such as poor nutrition) are more sensitive to the adverse effects of adult obesity on hypertension risk. Our study is the first to suggest that low birth weight might interact with adult obesity to increase hypertension risk in adult life. Targeting interventions and prevention of obesity, especially for those with low birth weight, might be given high priority.

Biological mechanisms by which low birth weight affects the development of hypertension are complex and remain equivocal. The proposed mechanisms are mostly related to reduced nephron counts, sympathetic hyperactivity, and impairment of vascular structure and function (17). In addition, intrauterine growth restriction may lead to impaired function of the hypothalamicpituitary-adrenal axis and increased activity of renin angiotensin aldosterone system, thereby elevating the risk for hypertension $(35,36)$. Animal studies have also found that fetal exposure to maternal protein restriction lead to an increase in renal inflammation, indicating an important role of inflammatory processes caused by low birth weight in the development of hypertension (37).

\section{STRENGTHS AND LIMITATIONS OF THE STUDY}

Our study has several strengths, including a large sample size, long-term follow-up, and strict adjustment for potential confounders. We also estimated the joint effects of birth weight 
TABLE 2 | Associations between birth weight and hypertension.

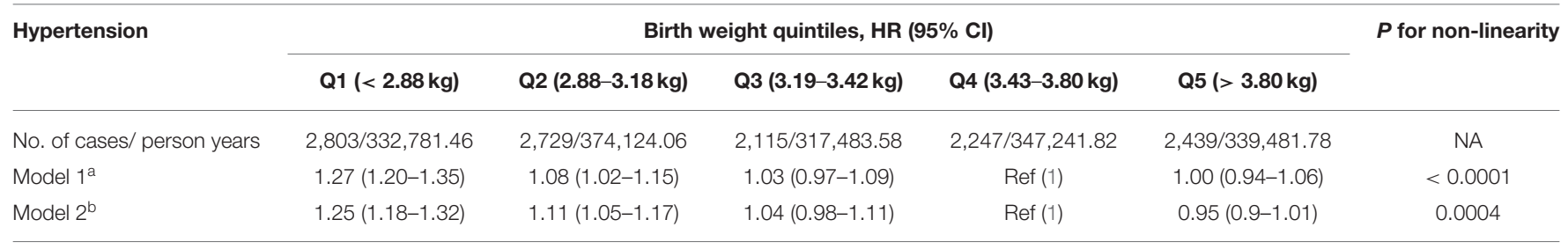

$H R$, hazard ratio; Cl, confidence interval; Ref, reference; NA, not applicable.

aModel 1: adjusted for age, sex, maternal smoking, breastfeeding, birth place, part of a multiple birth.

${ }^{b}$ Model 2: model 1 plus Townsend deprivation index, college or university degree, body mass index, physical activity (MET-hours/week), smoking status, alcohol intake frequency, intake

of vegetables and fruit, family history of hypertension, prevalent diabetes.

TABLE 3 | Associations between birth weight and blood pressure.

Blood pressure

Birth weight quintiles, $\beta$ coefficient $(95 \% \mathrm{Cl})$

Per SD increase

$\beta$ coefficient $(95 \% \mathrm{Cl})$

\begin{tabular}{|c|c|c|c|c|c|c|}
\hline & Q1 $(<2.88 \mathrm{~kg})$ & Q2 (2.88-3.18 kg) & Q3 (3.19-3.42 kg) & Q4 (3.43-3.80 kg) & Q5 (> 3.80 kg) & \\
\hline No. (\%) & 39,005 (19.53) & 43,754 (21.90) & $36,923(18.48)$ & $40,468(20.26)$ & $39,615(19.83)$ & NA \\
\hline \multicolumn{7}{|l|}{ SBP } \\
\hline Model $2^{b}$ & 2.36 (2.14 to 2.58$)$ & $1.23(1.01$ to 1.44$)$ & $0.72(0.5$ to 0.94$)$ & $\operatorname{Ref}(0)$ & $-0.69(-0.9$ to -0.47$)$ & $-1.07(-1.14$ to -1.00$)$ \\
\hline \multicolumn{7}{|l|}{ DBP } \\
\hline
\end{tabular}

Cl, confidence interval; Ref, reference; NA, not applicable; DBP, diastolic blood pressure; SBP, systolic blood pressure.

a Model 1: adjusted for age, sex, maternal smoking, breastfeeding, birth place, part of a multiple birth.

${ }^{b}$ Model 2: model 1 plus Townsend deprivation index, college or university degree, body mass index, physical activity (MET-hours/week), smoking status, alcohol intake frequency, intake of vegetables and fruit, family history of hypertension, prevalent diabetes. $S D=0.65$.

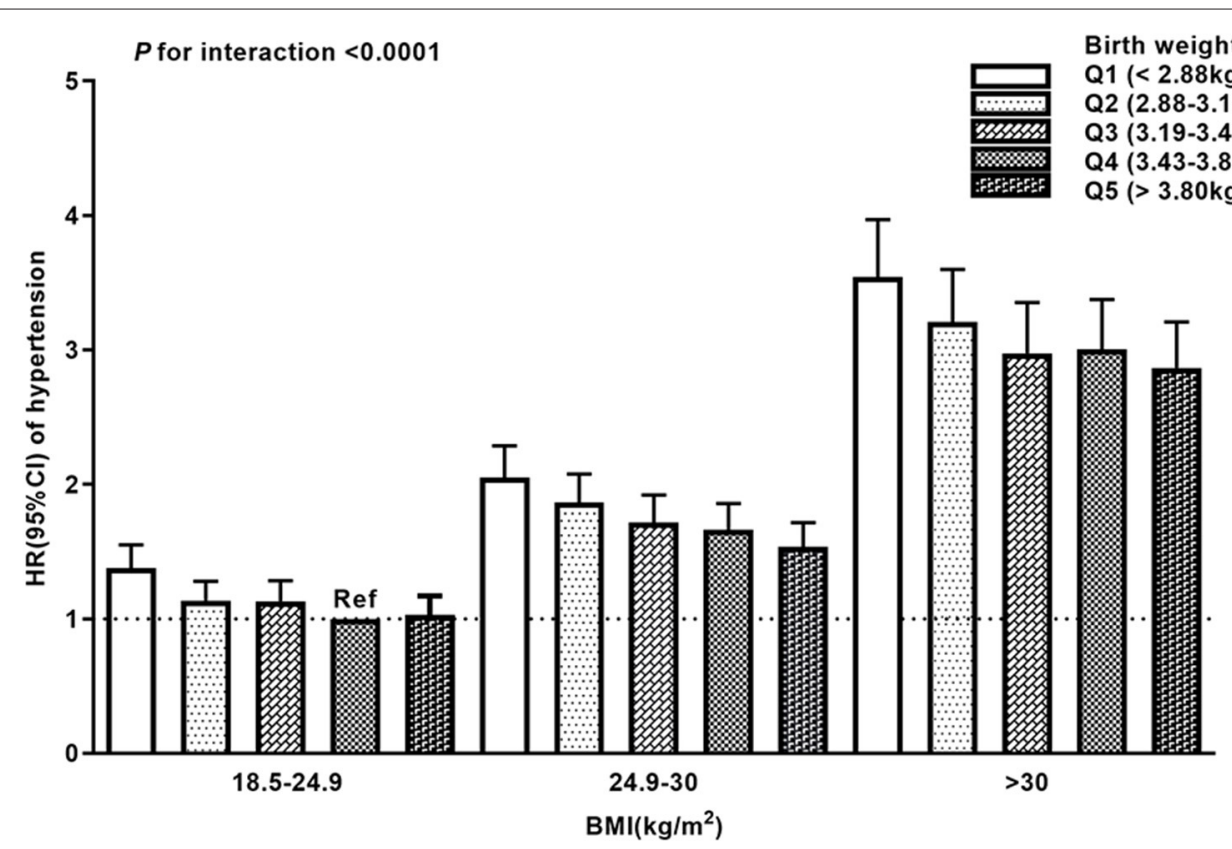

FIGURE 2 | Joint analysis of birth weight and body mass index (BMl) in the full model. The participants with birth weight in group Q4 (3.43-3.80 kg) and BMl in adult between $18.5-25.0 \mathrm{~kg} / \mathrm{m}^{2}$ were treated as the reference group (Ref). Error bars represent $95 \%$ confidence intervals of the hazard ratios. 
and adult obesity. Several limitations need to be acknowledged (12). First, early life exposures were based on self-reporting, which could lead to recall bias. However, we calculated the weighted Kappa correlation coefficients of self-reported birth weight between baseline and first $(n=12,171)$ and second follow-up $(n=4,272)$, which were 0.82 and 0.81 , respectively, indicating a good reliability of self-reported birth weight in the UK biobank. In addition, a previous study of the UK Biobank estimated the associations of self-reported birth weight with sex, deprivation index, multiple births, and maternal smoking, and supported the validity of self-reported birth weight (38). Some other studies also confirmed the validity of self-reported birth weight by comparing it with mothers' recall or hospital birth records $(39,40)$. Second, residual confounding could still exist in the observational study, and we were thus unable to make causal inferences. Finally, most of the UK Biobank participants were of caucasian race, which limits the generalizability of our findings to other ethnic populations.

\section{CONCLUSIONS}

The current study indicates a nonlinear inverse association between birth weight and hypertension and suggests birth weight between $3.43-3.80 \mathrm{~kg}$ as a threshold for reducing disease risk. Low birth weight may interact with adult obesity to increase hypertension risk in adult life. Further studies are warranted to explore underlying mechanisms.

\section{DATA AVAILABILITY STATEMENT}

The raw data supporting the conclusions of this article will be made available by the authors, without undue reservation.

\section{REFERENCES}

1. Miranda JO, Ramalho C, Henriques-Coelho T, Areias JC. Fetal programming as a predictor of adult health or disease: the need to reevaluate fetal heart function. Heart Fail Rev. (2017) 22:861-77. doi: 10.1007/s10741-017-9638-z

2. Lane RH. Fetal programming, epigenetics, and adult onset disease. Clin Perinatol. (2014) 41:815-31. doi: 10.1016/j.clp.2014.08.006

3. Chen W, Srinivasan SR, Berenson GS. Amplification of the association between birthweight and blood pressure with age: the Bogalusa Heart Study. $J$ Hypertens. (2010) 28:2046-52. doi: 10.1097/HJH.0b013e32833cd31f

4. Huxley R, Neil A, Collins R. Unravelling the fetal origins hypothesis: is there really an inverse association between birthweight and subsequent blood pressure? Lancet. (2002) 360:659-65. doi: 10.1016/S0140-6736(02)09834-3

5. Li Y, Ley SH, VanderWeele TJ, Curhan GC, Rich-Edwards JW, Willett WC, et al. Joint association between birth weight at term and later life adherence to a healthy lifestyle with risk of hypertension: a prospective cohort study. BMC Med. (2015) 13:175. doi: 10.1186/s12916-015-0409-1

6. Xia Q, Cai H, Xiang YB, Zhou P, Li H, Yang G, et al. Prospective cohort studies of birth weight and risk of obesity, diabetes, and hypertension in adulthood among the Chinese population. J Diabetes. (2019) 11:55-64. doi: 10.1111/1753-0407.12800

7. Andersson SW, Lapidus L, Niklasson A, Hallberg L, Bengtsson C, Hulthen L. Blood pressure and hypertension in middle-aged women in relation to weight and length at birth: a follow-up study. J Hypertens. (2000) 18:1753-61. doi: 10.1097/00004872-200018120-00008

\section{AUTHOR CONTRIBUTIONS}

$\mathrm{DH}, \mathrm{AG}$, and WZ contributed to the conception and design of the study. $\mathrm{YZ}$ and $\mathrm{DH}$ have full access to all the data in the study and take responsibility for the integrity of the data and the accuracy of the data analysis. YZ, JL, and QL did the statistical analysis and drafted the manuscript. XF and CX critically revised the manuscript for important intellectual content. All authors reviewed and approved the final manuscript.

\section{FUNDING}

This study was funded by the National Natural Science Foundation of China (81973127, 91839102, and 81722040), the National Key Research and Development Program of China (2019YFA0802701 and 2017YFC0908300), and the Science Foundation for Excellent Young Scholars of Jiangsu (BK20190083). The sponsors or funders had no involvements in any parts of this study. All authors confirm the independence of researchers from funding sources.

\section{ACKNOWLEDGMENTS}

We are grateful to the UK Biobank participants. This research has been conducted using the UK Biobank resource under application number 55858 .

\section{SUPPLEMENTARY MATERIAL}

The Supplementary Material for this article can be found online at: https://www.frontiersin.org/articles/10.3389/fcvm. 2021.637437/full\#supplementary-material

8. Pocobelli G, Dublin S, Enquobahrie DA, Mueller BA. Birth weight and birth weight for gestational age in relation to risk of hospitalization with primary hypertension in children and young adults. Matern Child Health J. (2016) 20:1415-23. doi: 10.1007/s10995-016-1939-7

9. Curhan GC, Chertow GM, Willett WC, Spiegelman D, Colditz GA, Manson JE, et al. Birth weight and adult hypertension and obesity in women. Circulation. (1996) 94:1310-5. doi: 10.1161/01.CIR.94.6.1310

10. Knop MR, Geng TT, Gorny AW, Ding R, Li C, Ley SH, et al. Birth weight and risk of type 2 diabetes mellitus, cardiovascular disease, and hypertension in adults: a meta-analysis of 7646267 participants from 135 studies. J Am Heart Assoc. (2018) 7:e008870. doi: 10.1161/JAHA.118.008870

11. Tian JY, Cheng Q, Song XM, Li G, Jiang GX, Gu YY, et al. Birth weight and risk of type 2 diabetes, abdominal obesity and hypertension among Chinese adults. Eur J Endocrinol. (2006) 155:601-7. doi: 10.1530/ eje. 1.02265

12. Bowers K, Liu G, Wang P, Ye T, Tian Z, Liu E, et al. Birth weight, postnatal weight change, and risk for high blood pressure among Chinese children. Pediatrics. (2011) 127:e1272-9. doi: 10.1542/peds.2010-2213

13. Kuciene R, Dulskiene V, Medzioniene J. Associations between high birth weight, being large for gestational age, and high blood pressure among adolescents: a cross-sectional study. Eur J Nutr. (2018) 57:373-81. doi: 10.1007/s00394-016-1372-0

14. Johnsson IW, Haglund B, Ahlsson F, Gustafsson J. A high birth weight is associated with increased risk of type 2 diabetes and obesity. Pediatr Obes. (2015) 10:77-83. doi: 10.1111/ijpo.230 
15. Wei JN, Li HY, Sung FC, Lin CC, Chiang CC, Li CY, et al. Birth weight correlates differently with cardiovascular risk factors in youth. Obesity. (2007) 15:1609-16. doi: 10.1038/oby.2007.190

16. Seravalle G, Grassi G. Obesity and hypertension. Pharmacol Res. (2017) 122:1-7. doi: 10.1016/j.phrs.2017.05.013

17. Alexander BT, Dasinger JH, Intapad S. Fetal programming and cardiovascular pathology. Comprehen Physiol. (2015) 5:997-1025. doi: 10.1002/cphy. c140036

18. Tian J, Qiu M, Li Y, Zhang X, Wang H, Sun S, et al. Contribution of birth weight and adult waist circumference to cardiovascular disease risk in a longitudinal study. Sci Rep. (2017) 7:9768. doi: 10.1038/s41598-017-1017 6-6

19. Lawlor DA, Ronalds G, Clark H, Smith GD, Leon DA. Birth weight is inversely associated with incident coronary heart disease and stroke among individuals born in the 1950s: findings from the Aberdeen Children of the 1950s prospective cohort study. Circulation. (2005) 112:1414-8. doi: 10.1161/CIRCULATIONAHA.104.528356

20. Collins R. What makes UK Biobank special? Lancet. (2012) 379:1173-4. doi: 10.1016/S0140-6736(12)60404-8

21. Honigberg MC, Patel AP, Lahm T, Wood MJ, Ho JE, Kohli P, et al. Association of premature menopause with incident pulmonary hypertension: a cohort study. PLoS ONE. (2021) 16:e247398. doi: 10.1371/journal.pone.0247398

22. Sudlow C, Gallacher J, Allen N, Beral V, Burton P, Danesh J, et al. UK biobank: an open access resource for identifying the causes of a wide range of complex diseases of middle and old age. PLoS Med. (2015) 12:e1001779. doi: 10.1371/journal.pmed.1001779

23. Tan M, Cai L, Ma J, Jing J, Ma Y, Chen Y. The association of gestational age and birth weight with blood pressure among children: a Chinese national study. $J$ Hum Hypertens. (2018) 32:651-9. doi: 10.1038/s41371-018-0084-8

24. Dong YH, Zou ZY, Yang ZP, Wang ZH, Jing J, Luo JY, et al. Association between high birth weight and hypertension in children and adolescents: a cross-sectional study in China. J Hum Hypertens. (2017) 31:737-43. doi: $10.1038 /$ jhh.2017.22

25. Blencowe H, Krasevec J, de Onis M, Black RE, An X, Stevens GA, et al. National, regional, and worldwide estimates of low birthweight in 2015, with trends from 2000: a systematic analysis. Lancet Global Health. (2019) 7:e849-e60. doi: 10.1016/S2214-109X(18)30565-5

26. Kramer MS. Determinants of low birth weight: methodological assessment and meta-analysis. Bull World Health Org. (1987) 65:663-737.

27. Barker DJ. The developmental origins of chronic adult disease. Acta Paediatr Suppl. (2004) 93:26-33. doi: 10.1111/j.1651-2227.2004.tb00236.x

28. Bustos P, Amigo H, Bangdiwala SI, Pizarro T, Rona RJ. Does the association between birth weight and blood pressure increase with age? A longitudinal study in young adults. J Hypertens. (2016) 34:1062-7. doi: 10.1097/HJH.0000000000000912

29. Hovi P, Vohr B, Ment LR, Doyle LW, McGarvey L, Morrison KM, et al. Blood pressure in young adults born at very low birth weight: adults born preterm international collaboration. Hypertension. (2016) 68:880-7. doi: 10.1161/HYPERTENSIONAHA.116.08167
30. Warrington NM, Beaumont RN, Horikoshi M, Day FR, Helgeland $\varnothing$, Laurin C, et al. Maternal and fetal genetic effects on birth weight and their relevance to cardio-metabolic risk factors. Nat Genet. (2019) 51:804-14. doi: 10.1038/s41588-019-0403-1

31. Hardy R, Wadsworth ME, Langenberg C, Kuh D. Birthweight, childhood growth, and blood pressure at 43 years in a British birth cohort. Int $J$ Epidemiol. (2004) 33:121-9. doi: 10.1093/ije/dyh027

32. Järvelin MR, Sovio $U$, King V, Lauren L, Xu B, McCarthy MI, et al. Early life factors and blood pressure at age 31 years in the 1966 northern Finland birth cohort. Hypertension. (2004) 44:838-46. doi: 10.1161/01.HYP.0000148304.33869.ee

33. Lurbe E, Carvajal E, Torro I, Aguilar F, Alvarez J, Redon J. Influence of concurrent obesity and low birth weight on blood pressure phenotype in youth. Hypertension. (2009) 53:912-7. doi: 10.1161/HYPERTENSIONAHA.109.129155

34. Leon DA, Koupilova I, Lithell HO, Berglund L, Mohsen R, Vagero D, et al. Failure to realise growth potential in utero and adult obesity in relation to blood pressure in 50 year old Swedish men. BMJ. (1996) 312:401-6. doi: 10.1136/bmj.312.7028.401

35. Rasyid H, Bakri S. Intra-uterine growth retardation and development of hypertension. Acta Med Indones. (2016) 48:320-4. https://pubmed.ncbi.nlm. nih.gov/28143994/

36. Seckl JR. Prenatal glucocorticoids and long-term programming. Eur J Endocrinol. (2004) 151 (Suppl 3):U49-62. doi: 10.1530/eje.0.151u049

37. Lurbe E, Ingelfinger J. Developmental and early life origins of cardiometabolic risk factors: novel findings and implications. Hypertension. (2021) 77:308-18. doi: 10.1161/HYPERTENSIONAHA.120.14592

38. Tyrrell JS, Yaghootkar H, Freathy RM, Hattersley AT, Frayling TM. Parental diabetes and birthweight in 236030 individuals in the UK biobank study. Int J Epidemiol. (2013) 42:1714-23. doi: 10.1093/ije/dyt220

39. Troy LM, Michels KB, Hunter DJ, Spiegelman D, Manson JE, Colditz GA, et al. Self-reported birthweight and history of having been breastfed among younger women: an assessment of validity. Int J Epidemiol. (1996) 25:122-7. doi: 10.1093/ije/25.1.122

40. Walton KA, Murray LJ, Gallagher AM, Cran GW, Savage MJ, Boreham C. Parental recall of birthweight: a good proxy for recorded birthweight? Eur J Epidemiol. (2000) 16:793-6. doi: 10.1023/A:1007625030509

Conflict of Interest: The authors declare that the research was conducted in the absence of any commercial or financial relationships that could be construed as a potential conflict of interest.

Copyright (C) 2021 Zhang, Liang, Liu, Fan, Xu, Gu, Zhao and Hang. This is an open-access article distributed under the terms of the Creative Commons Attribution License (CC BY). The use, distribution or reproduction in other forums is permitted, provided the original author(s) and the copyright owner(s) are credited and that the original publication in this journal is cited, in accordance with accepted academic practice. No use, distribution or reproduction is permitted which does not comply with these terms. 